\title{
Hidradenitis suppurativa
}

\section{R. Gary Sibbald MD, Asfandyar Mufti BMSc}

A 43-year-old man who was a current smoker was referred to a wound healing clinic for recurrent painful sacral pilonidal abscesses (Figure 1). Multiple abscesses, sinus tracts, scar tissue and open pits were seen in the patient's sacral coccyx and gluteal region (Figure 1). The abscesses had first appeared when the was about 20 years old, and multiple surgical procedures had been done to drain the abnormally thick exudate. Based on the sacral location, presence of multiple sinus tracts, scarring and paired open pits, we diagnosed hidradenitis suppurativa.

Hidradenitis suppurativa is a chronic, recurrent, inflammatory disease of intertriginous skin (axillary, inguinal, genital and perineal), mostly affecting women. ${ }^{1}$ The prevalence is about $1 \%,{ }^{2}$ and $30 \%$ of patients with the condition have a family history. ${ }^{3}$ It is uncommon before puberty or in older adults. ${ }^{2,4}$ The pathophysiology involves occlusion of the hair follicle, with secondary involvement of apocrine glands.

Hidradenitis suppurativa is often misdiagnosed as recurrent pilonidal sinus, furuncles, carbuncles or abscesses. The differential diagnosis includes cutaneous Crohn disease. ${ }^{2}$ It is strongly associated with smoking and obesity, which may exacerbate it. ${ }^{5}$ The diagnosis is made based on a history of recurrent painful suppurative abscesses, nodules or cysts (> 2 lesions in $6 \mathrm{mo}$ ) in flexural locations, with scarring sinus tracts and paired open pits. ${ }^{2}$

The European Academy of Dermatology and Venereology guideline recommends weight reduction and smoking cessation, when appropriate, for patients with hidradenitis suppurativa. ${ }^{2}$ Mild disease with a limited number of lesions can be managed with local incision and drainage, topical clindamycin and courses of oral antibiotics (e.g., tetracyclines, rifampin, clindamycin). More persistent localized disease with early scarring may require intralesional steroid injections, removal of scar tissue and systemic acitretin. ${ }^{2}$ Advanced disease with extensive scarring requires wide surgical excision with healing by secondary intention or rotational flaps. Randomized controlled trials of biologic agents with anti-tumour necrosis factor properties have

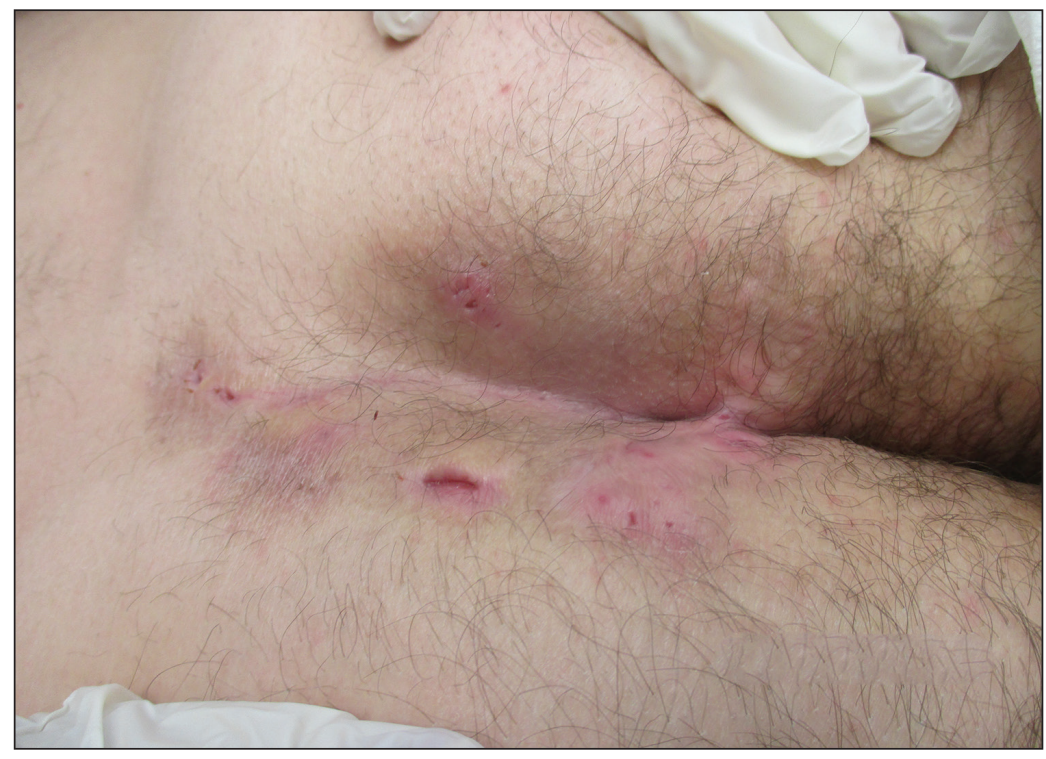

Figure 1: Nodules, active inflamed abscesses, paired pits from previously inflamed hair follicles and scarring tracts in the sacral coccyx and gluteal region of a 43-year-old man with hidradenitis suppurativa.

shown them to be effective in cases resistant to treatment. $^{2}$

Our patient was given topical clindamycin (combined with benzoyl peroxide), intralesional steroid injections and oral doxycycline (100 mg) twice daily. After six weeks, the lesions showed improvement, with minimal pain and discharge from the abscesses.

Patient quality of life may be improved with early diagnosis, referral to a dermatologist or surgeon, and an interprofessional approach to care.

\section{References}

1. Shlyankevich J, Chen AJ, Kim GE, et al. Hidradenitis suppurativa is a systemic disease with substantial comorbidity burden: a chart-verified case-control analysis. J Am Acad Dermatol 2014; $71: 1144-50$

2. Zouboulis CC, Desai N, Emtestam L, et al. European S1 guideline for the treatment of hidradenitis suppurativa/acne inversa. $J$ Eur Acad Dermatol Venereol 2015;29:619-44.

3. Micheletti RG. Hidradenitis suppurativa: current views on epidemiology, pathogenesis, and pathophysiology. Semin Cutan Med Surg 2014;33(Suppl 3)S48-50.

4. Mengesha YM, Holcombe TC, Hansen RC. Prepubertal hidradenitis suppurativa: two case reports and review of the literature. Pediatr Dermatol 1999;16:292-6.

5. Alikhan A, Lynch PJ, Eisen DB. Hidradenitis suppurativa: a comprehensive review. J Am Acad Dermatol 2009;60:539-61.
Competing interests: None declared.

This article has been peer reviewed.

The authors have obtained patient consent.

Affiliations: Division of Dermatology (Sibbald), Dalla Lana School of Public Medicine, Faculty of Medicine and Faculty of Public Health, University of Toronto, Toronto, Ont.; Faculty of Medicine (Mufti) University of Ottawa, Ottawa, Ont.

Correspondence to: R. Gary Sibbald, gary. sibbald@utoronto.ca

CMAJ 2015. DOI:10.1503 /cmaj.150018 Health, Department of 